\title{
Riots - Zur Verortung eines unscharfen Phänomens
}

\author{
Janna Frenzel, Philippe Greif,
} Fabian Klein und Sarah Uhlmann

In den letzten Jahren berichteten die Medien immer wieder von riots, gewaltvollen Ausschreitungen, die sich in den Großstädten Europas und Nordamerikas ereigneten: in Frankreich (2005), in Griechenland (2008/2011), in England (2011), in Istanbul und Stockholm (2013) und Ferguson (2014/2015), aber auch im letzten Jahr in Kiew (2015). Auch in Deutschland lieferten sich Demonstrant_innen bei den Blockupy Protesten in Frankfurt (2015) heftige Auseinandersetzungen mit den Ordnungskräften.

Wir haben den Eindruck, dass es in verschiedenen Sprachen keine einheitliche Begrifflichkeit für das gibt, was umgangssprachlich unter dem englischen Begriff riot verhandelt wird. Zudem scheint nach wie vor unklar, welche Phänomene warum als riots bezeichnet bzw. nicht bezeichnet werden. Der riot-Begriff hat sich in den letzten Jahren auch zunehmend unter das sozialwissenschaftliche Vokabular gemischt, ohne dass dies mit einer entsprechenden Konzeptionierung einher gegangen wäre. Zwar gibt es sozialwissenschaftliche Arbeiten zum Thema, auf die wir im Folgenden noch genauer eingehen werden, eine systematische Untersuchung im Rahmen der Protestforschung steht jedoch noch aus. In der breiten Öffentlichkeit findet tendenziell nur eine oberflächliche Auseinandersetzung mit riots statt. Nach den Aufständen in Frankreich (2005) und in England (2011) wurden die Teilnehmenden in den Medien überwiegend als Kriminelle verurteilt und stigmatisiert, wodurch den riots jeglicher politischer Charakter abgesprochen wurde (siehe die Beiträge zu England und Frankreich in diesem Heft).

Bereits 1979 haben Piven und Cloward darauf hingewiesen, dass sich die Möglichkeiten zum Protest für verschiedene Gruppen in kapitalistischen Gesellschaften fundamental voneinander unterscheiden. Marginalisierte Gruppen hätten ihrer Ansicht nach nur sehr begrenzt Zugang zu institutionalisierter Macht und seien gewissermaßen gezwungen, disruptive action und collective defiance als Aktionsformen einzusetzen, um ihre Unzufriedenheit zu artikulieren. Es lohne sich für sie nicht, auf formalisiertem Wege (beispielsweise durch Wahlen oder Petitionen) Forderungen zu stellen, da dies keine substantiellen Veränderungen ihrer Lebensumstände bewirken könne. 
Der Einordnung von riots als Protestverhalten schließen sich viele Sozialwissenschaftler_innen an, denn schließlich stellen riots gültige gesellschaftliche Regeln und Normen deutlich infrage. Eine systematische Analyse und Ursachenforschung wird dabei jedoch nur selten durchgeführt. Der begrifflichen Unschärfe des Begriffs steht die Schwierigkeit einer genaueren Bestimmung dessen, was riots sind, gegenüber. Einerseits verstellt seine polarisierende, entweder ablehnende oder zustimmende, Verwendung eine offene Auseinandersetzung. Andererseits erschwert die Überladung des Begriffes, der für unterschiedlichste Situationen gleichermaßen herangezogen wird, seine Verwendung als Analysekategorie.

Das Anliegen des vorliegenden Themenheftes ist es, der öffentlich vorherrschenden Entdifferenzierung von riots theoretische Analysen entgegenzusetzen, in denen Akteur_innen, Ziele und gesellschaftliche Verhältnisse, in denen riots stattfinden, untersucht werden und nicht zuletzt auch nach dem politischen Gehalt von riots gefragt wird. Davon auszugehen, dass alle riots per se progressiv sind, wäre sicher falsch. Fatal ist es jedoch, wenn auf riots vorrangig ordnungspolitisch und repressiv reagiert wird, anstatt sie zum Anlass zu nehmen, gesellschaftliche Machtverhältnisse und soziale Ungleichheit kritisch in den Blick zu nehmen. Sinnvoll erscheint uns deshalb die Konzeptionierung von riots als „kollektive Bearbeitung der asymmetrisch stabilisierten Machtbeziehungen“, wie sie Julika Mücke und Moritz Rinn in diesem Heft vornehmen. Um an das Thema riots heranzuführen, möchten wir in dieser Einleitung zuerst auf die Analyseperspektive der Sozialen Bewegungsforschung sowie auf die Verwendung des Begriffs riot eingehen, bevor wir anschließend zwei Aspekte von riots herausgreifen, die wir in der Forschung als besonders zentral erachten: die Gewalt und der politische Gehalt.

\section{Riots im Kontext der Sozialen Bewegungsforschung}

Obwohl Ereignisse, die riot genannt werden, regelmäßig zum Gegenstand wissenschaftlicher Auseinandersetzungen werden, hat sich bislang kein eigenständiges Forschungsfeld etablieren können. Vielmehr beschäftigen sich mit der Erforschung solcher Ereignisse Wissenschaftler_innen verschiedener Disziplinen, die ihre je eigenen Erkenntnisinteressen mit je eigenen Kategorien verfolgen, seien diese aus der Bewegungs- oder Protestforschung, der Stadt- bzw. Raumsoziologie oder der Gewaltsoziologie.

Die traditionelle Soziale Bewegungsforschung, in der riots als Forschungsgegenstand wohl am ehesten anzusiedeln wären, tut sich gerade im deutschen Forschungskontext schwer, riots als einen ihrer Untersuchungsgegenstände zu fassen. Das liegt zum einen an der verbreiteten Überzeugung, in Deutschland gäbe es keine - etwa mit den banlieue-Aufständen in Frankreich (2005) oder den riots in England (2011) - vergleichbaren Phänomene (vgl. dazu den Beitrag von Mücke und Rinn in diesem Heft). Zum anderen ist die Schwierigkeit, den Gegenstand zu fassen, aber auch einer spezifischen Perspektive und Kategorienbildung geschuldet.[1]

Soziale Bewegungen werden oft als kollektive Akteur_innen begriffen, die zielgerichtet und vor dem Hintergrund strategischer Überlegungen Einfluss auf die institutionalisierte Politik zu nehmen versuchen, um 
sozialen Wandel zu erreichen (oder zu verhindern). Dabei bedienen sie sich bestimmter Aktionsformen wie Demonstrationen, Medienstatements und Versammlungen, um ihre politischen Forderungen öffentlich sicht- und hörbar zu machen (vgl. Tilly/Wood 2013: 8). Zwar erkennt die Soziale Bewegungsforschung an, dass gewaltsames Handeln eine mögliche Aktionsform sein kann. Wo sie aber mit einem Handeln konfrontiert ist, ohne dass sich zumindest ein informelles Netzwerk, eine Gruppenidentität und eine klare Gegnerschaft ausmachen lassen (vgl. della Porta/Diani 1999: 20) beziehungsweise wo sich politische Forderungen und Botschaften nicht unmittelbar aus Bewegungsdokumenten, Flyern, Postern und Transparenten erschließen, gerät sie häufig an ihre Grenzen.[2] Zwar wird das Geschehen auf der Straße teilweise auch als unorganisierter, spontaner und emotionaler Ausdruck der Unzufriedenheit mit gesellschaftlichen Verhältnissen interpretiert. Es scheinen diesem Handeln aber die Dauerhaftigkeit und Verstetigung, die Gerichtetheit auf eine gesellschaftliche Transformation und der an die institutionalisierte Politik appellierende Charakter wie auch das Ziel eines politischen Machtwechsels zu fehlen; also die Voraussetzungen, die es als Gegenstand der Sozialen Bewegungsforschung qualifizieren würden. So riskiert die Soziale Bewegungsforschung, riots als „sinnlose Gewalt“ abzutun, die sich zwar an gesellschaftlichen Missständen entzündet, selbst aber keine politisch motivierte Handlungsform darstellen kann (vgl. Dikeç 2004: 191).

Häufig wird versucht, riots - ebenso wie soziale Bewegungen - allein nach formalen Kriterien zu fassen (vgl. den Beitrag von Dieter Rucht in diesem Heft). Dabei bleibt, wie Rucht selbst anmerkt, „die thematische Seite unbestimmt“. Als zentrale Merkmale, um riots von sozialen Bewegungen zu unterscheiden, dienen dann die beim riot (tatsächlich oder scheinbar) fehlende Artikulation eines gemeinsamen Ziels, fehlende zielgerichtete strategische Überlegungen sowie ein geringer Organisationsgrad.

Keine dieser Bestimmungen scheint jedoch durchgängig auf alle als riot gelabelten Phänomene zuzutreffen: Vielfach formulieren rioter durchaus übereinstimmende Motive und Ziele ihres Handelns, wenn sie danach gefragt werden. Beispielsweise nennen in einer Studie 85 Prozent der interviewten rioter Ärger und Frustration über das alltägliche Polizeiverhalten, vor allem stop-and-search-Praktiken, als „,wichtigen“ oder „,sehr wichtigen“ Grund für die English riots (vgl. Guardian/LSE 2011: 4). Wenn der Blick auf subkulturelle Ausdrucksformen wie etwa Graffiti und Musik erweitert wird, scheint die Vorstellung, es gäbe keine verbindenden Überzeugungen und Ziele, kaum noch haltbar (vgl. für Frankreich RIAD 2012 und die Beiträge in Mennel/ Nowotny 2014 sowie den Beitrag von Jobard und Greif in diesem Heft). Wenn es über soziale Medien einerseits zu (massenmedial skandalisierten) Koordinierungen von riots kommt und in Internetforen unter anderem Teilnehmende die riots diskutieren, muss auch die Vorstellung einer völlig abwesenden Organisation und Strategie als Unterscheidungsmerkmal zu sozialen Bewegungen relativiert werden. Unter Verweis auf Netzwerke (freundschaftliche, familiäre oder durch die community begründete) und den Einsatz von Mobiltelefonen oder Blogs zum Aufbau einer losen Protestinfrastruktur kommen auch Donatella della Porta und Bernard Gbikpi zu dem Schluss: „[R]iots haben einen höheren Organisationsgrad als gemeinhin anerkannt“ (2012: 89f.). In diesem Sinne weist Dieter Rucht in diesem Heft ganz richtig 
darauf hin, dass „riots durchaus im Kontext einer sozialen Bewegung vorkommen oder auch deren Ausgangspunkt bilden"können. Auch Frances Fox Piven (2012: 27) spricht in diesem Sinne davon, dass Gewalt und die Androhung von Gewalt auf komplexe Weise mit den Anstrengungen sozialer Bewegungen verwoben seien, Macht auszuüben.

Auch andere Beiträge des Themenheftes fragen auf unterschiedliche Weise nach dem Verhältnis von riots und sozialen Bewegungen, ohne dass sich eine allgemeinverbindliche Definition herauskristallisieren würde. Dies kann auch nicht Ziel der Auseinandersetzung sein. Es kann weder darum gehen, eine strikte, in jedem Fall gültige Abgrenzung von riots zu sozialen Bewegungen zu formulieren, noch darum, eine Unterscheidung vollständig aufzugeben: Nicht alle riots lassen sich notwendigerweise als (Teil) soziale(r) Bewegungen beschreiben, und nicht in jeder sozialen Bewegung sind riots Bestandteil eines Repertoires an Aktionsformen. Vielmehr scheint es geboten, in jedem Einzelfall danach zu fragen, von wem, in welchem Kontext, wie und aus welchen Gründen ein spezifisches Ereignis oder Phänomen das Label riot erhält und wie es um das Verhältnis des so Bezeichneten zu sozialen Bewegungen steht.

Eine Möglichkeit riots, soziale Bewegungen und andere friedlich oder gewaltsam verlaufende politische Auseinandersetzungen gemeinsam zu erfassen, bieten Tilly und Tarrow (2015) mit ihrem Konzept von contentious politics. Für Tilly und Tarrow bedeutet dies keineswegs eine Gleichsetzung von sozialen Bewegungen mit riots, im Gegenteil sprechen sie sich explizit gegen eine Ausweitung des Bewegungsbegriffs aus (ebd.: 11). Im Zusammenkommen von contention, politics und collective action sehen sie jedoch eine Gemeinsamkeit sozialer Bewegungen mit anderen Protestformen, wie beispielsweise mit riots. Dieses Gemeinsame bestehe in einem interaktiven, kollektiven Aufstellen von Forderungen, das sich auf die Interessen anderer Akteur_innen auswirke und in dem Regierungen als Anspruchsteller, -adressaten oder Dritte involviert seien (ebd.: 21), auch wenn sich die konkreten Handlungsweisen und Aktionsformen unterscheiden. Riots würden dann als eine konkrete Ausformung von contentious politics fassbar, sodass Seferiades und Johnston sich zurecht fragen, ob gewaltsamer Protest vielleicht die Art und Weise sei, wie sich contentious politics in Krisenzeiten wandele (2012a: 3).

\section{Riot - ein diffuser Begriff zwischen Recht, Umgangssprache und Wissenschaft}

Riots als Form von contentious politics zu fassen, kommt ihrem Wesen zwar vermutlich näher, aber es klärt immer noch nicht abschließend, wovon wir eigentlich sprechen, wenn wir das Wort riot benutzen. Ursprünglich ist die englische Bezeichnung riot ein juristischer Begriff, der Teil der Umgangssprache geworden ist und sich medial durchgesetzt hat. Betrachtet man eine juristische Definition aus Großbritannien, so stellt ein riot nach dem Public Order Act von 1986 folgenden Straftatbestand dar: Eine Gruppe von zwölf oder mehr Personen droht oder wendet absichtlich gesetzeswidrige Gewalt an, sodass anwesende Personen Grund haben, sich um ihre persönliche Sicherheit zu sorgen.[3] 
Im Deutschen fände die Bezeichnung riot wohl am ehesten ihre Entsprechung in Begriffen wie „Aufruhr“, „Ausschreitung“, „Aufstand“ oder „Krawall“. Im juristischen Kontext finden sich historische Definitionen für den Begriff „Aufruhr“. Paragraph 115 des Reichsstrafgesetzbuches von 1871 regelte, dass „wer an einer öffentlichen Zusammenrottung, bei welcher eine der in den $\S 113$ und 114 bezeichneten Handlungen mit vereinten Kräften begangenen wird, Theil nimmt, [...] wegen Aufruhrs mit Gefängniß nicht unter sechs Monaten bestraft [wird]“[4]. Die genannten Paragraphen beziehen sich auf Vergehen, die die Tatbestände „Widerstand gegen die Staatsgewalt“ bzw. „Störung von Amtshandlungen“ betreffen.

Während die angeführte britische Definition das Augenmerk auf das Thema „Sicherheit“ richtet, steht in der (historischen) deutschen Definition im Zentrum, dass sich ein „Aufruhr“ gegen den Staat und seine Amtsträger_ innen richtet. Bemerkenswert ist, dass im britischen Gesetzestext bereits die Androhung von Gewalt Teil des Straftatbestandes ist. Ebenso fällt auf, dass von einer "gemeinsamen Absicht“ (common purpose) die Rede ist, auf die durch das Verhalten der Beteiligten geschlossen werden könne (Paragraph 1(2)). Dies hat sich allerdings nicht im wissenschaftlichen Gebrauch des Begriffes niedergeschlagen, insofern vor allem in der Sozialen Bewegungsforschung davon ausgegangen wird, dass rioter gerade kein explizites gemeinsames Anliegen haben und riots damit auch keine zielgerichteten Handlungen darstellen.

Jenseits einer Betrachtung der juristischen Begriffsverwendung stellt sich die Frage, welches Phänomen oder welche Phänomene der Begriff riot eigentlich fassen soll. Die sozialwissenschaftliche Beschäftigung mit riots bildet kein abgegrenztes (vielleicht auch kein abgrenzbares) Forschungsfeld und ist keineswegs kanonisiert. Der Begriff riot wird in der einschlägigen Literatur häufig verwendet, ohne dass er analytisch näher bestimmt oder von verwandten Begriffen abgegrenzt wird. Das Phänomen, das mit ihm beschrieben wird, wird meist nur implizit definiert, indem bestimmte Ereignisse und/oder Aktionsformen als riots bezeichnet werden. Oder es wird einfach vorausgesetzt, dass es sich bei bestimmten empirischen Fallbeispielen um riots handelt. Dies lässt sich am Beispiel der riots in England 2011 zeigen: So nimmt etwa Tom Slater in seinem Aufsatz „From ,Criminality“ to Marginality: Rioting Against a Broken State“ (2011) als gegeben an, dass es sich bei den Straßenkämpfen und Plünderungen um riots gehandelt hat. Ohne den Begriff zu klären, beginnt er, die politische Bedeutung dieser riots zu untersuchen. Andere Autor_innen wechseln relativ wahllos zwischen Alternativbegriffen wie civil unrest oder violent urban disturbance hin und her (vgl. Beiträge in Waddington/Jobard/King 2009). Oder sie greifen auf analytische Konzepte aus anderen Feldern zurück. So forderte Michael Keith (1993), riots mithilfe von Konzepten wie local history (lokale Geschichte) und spatiality (Räumlichkeit) zu analysieren. Er kritisiert, dass die Besonderheiten einzelner riots in Verallgemeinerungen verloren gingen und riot eben nicht gleich riot sei. Auch neuere Ansätze weisen darauf hin, dass riots nicht aus einem geschlossenen Handlungsset bestehen, sondern an verschiedenen Orten und in verschiedenen Kontexten unterschiedliche Formen annehmen können. Phillips, Frost und Singleton (2013) beschreiben die riots in England 2011 als disturbances (Störungen), die sich von Demonstrationen gegen die 
Polizei in gewaltsame Ausschreitungen (einschließlich Plünderungen und Vandalismus) transformiert hätten. Jenseits solcher kritischen Hinweise gibt es jedoch wenige Versuche, die definitorische Lücke zu füllen.

Einen Versuch, riots zu kategorisieren und voneinander abzugrenzen, unternahm Gary Marx bereits im Jahre 1970. Als zentrale Analysekriterien bestimmt er zum einen, ob es unter den riotern eine geteilte Überzeugung gibt, und zum anderen, ob ein riot geeignet ist, das Problem zu lösen, aufgrund dessen eine Gruppe an ihm teilnimmt. Anhand dieser Kriterien unterscheidet er drei Arten von riots: Erstens instrumentelle riots, bei denen eine gemeinsame Überzeugung vorhanden ist (als Beispiele werden food riots und riots in Fabriken genannt); zweitens riots, bei denen es zwar eine geteilte Überzeugung gibt, die aber nicht instrumentell die Probleme einer Gruppe lösen (angeführte Beispiele sind Pogrome und sogenannte communal riots); drittens issueless riots, bei denen die Teilnehmenden keine Überzeugung teilen und die keinerlei Auswirkungen auf einen sozialen Wandel haben (vgl. Marx 1970: 21). Eine solche Unterscheidung ist sicherlich ein lohnenswerter Versuch, unterschiedliche Phänomene voneinander abzugrenzen, die als riots gelten oder als solche bezeichnet werden. Ob es jedoch issueless riots überhaupt gibt, ist zu bezweifeln. Marx nimmt an, dass es zu gewaltsamen riots kommen kann, ohne dass die Teilnehmenden eine bestimmte Motivation für ihre Handlungen haben - und geht aus unserer Sicht fehl in der Annahme, dass der politische Gehalt von riots immer klar ersichtlich sein muss oder sich aus dem unmittelbaren Kontext (beispielsweise bei einem Streik in einer Fabrik) erschließen lässt.

Die neuere Forschung zu riots nimmt vor allem soziale Ungleichheiten in den Blick. Sie beschäftigt sich mit der sozialen und räumlichen Trennung von Arm und Reich im urbanen Raum, zunehmender Prekarisierung durch Prozesse neoliberaler Umstrukturierung (Arbeitslosigkeit, Rückgang staatlich finanzierter Sozialpolitik etc.), der rassistischen Stigmatisierung von Minderheiten und -allgemeiner gesprochen - der postkolonialen Situation in europäischen Metropolen.

Um riots besser zu verorten und uns dem Phänomen trotz oder gerade wegen der unklaren Begrifflichkeit zu nähern, greifen wir im Folgenden zwei zentrale Aspekte der wissenschaftlichen Debatte um riots heraus: Erstens die Gewaltförmigkeit und zweitens den politischen Gehalt von riots. Dadurch wollen wir nicht nur einige der Linien nachzeichnen, an denen sich die Forschung zu riots bisher orientiert hat, sondern hoffen gleichzeitig, mehr Klarheit über den Forschungsgegenstand selbst zu erzeugen.

\section{Zum Verhältnis von Gewalt und riots}

Das Sprechen über riots - in den Medien wie in der Forschung - ist geprägt durch ein Sprechen über die Gewalt, die in ihnen zum Ausdruck kommt. Der Gewaltaspekt ist entscheidend dafür, ob ein Geschehen überhaupt erst als riot in den Blick kommt. Es gibt natürlich Gewalt abseits von riots, umgekehrt aber wird der Begriff riot erst dann in Anschlag gebracht, wenn eine Auseinandersetzung durch kollektive physische Gewalt geprägt ist. Gerade diese Gewalt ist es, die rioting von anderen Formen kollektiven Handelns im öffentlichen Raum (etwa zivilem Ungehorsam) unterscheidet 
bzw. die für diese Unterscheidung relevant gemacht wird und entsprechende Repressionen nach sich zieht. Das dynamische Wechselspiel von Provokation und Reaktion zwischen Teilnehmenden von riots und der Polizei stellt eine typische Verlaufsform eines riots dar. Sie ist teilweise in einer solchen Form ritualisiert, dass sie von beiden Akteuren geradezu erwartet wird.

Die von riotern ausgehende Gewalt richtet sich dabei typischerweise einerseits gegen Dinge, wobei diese „gegen Dinge gerichtete Gewalt“vor allem medial häufig zur „Gewalt gegen Menschen“ umgedeutet wird, indem auf die Schädigung von Eigentümer_innen (von Geschäften, Autos etc.) hingewiesen wird. Andererseits sind riots durch gewaltsame Auseinandersetzungen zwischen riotern und der Polizei charakterisiert. Die Ordnungskräfte werden dabei vor allem als Repräsentant_innen der gesellschaftlichen Verhältnisse wahrgenommen. Die Ablehnung dieser Verhältnisse als soziale Ausschlüsse produzierende und aufrechterhaltende Wirklichkeit (durch die Aufständischen) kann wohl als Gemeinsamkeit der allermeisten als riot gelabelten Phänomene gelten. Gerade im Kontext der riots in Frankreich 2005 oder in England 2011 ist die symbolisch-repräsentative Funktion der Polizei - als Ziel von Gewalt - nicht zu trennen von einem konfliktiven Alltag der rioter, in dem die Polizei vor allem als Instanz allgegenwärtiger Schikanen in Form von racial profiling und stop-and-search-Maßnahmen wahrgenommen und erlebt wird (Altenried 2012: 19ff., vgl. auch den Beitrag von Lollia, Meftach und Greif in diesem Heft sowie das sub \urban-Themenheft „Illegalität - Stadt - Polizei“ 2014).

In der weitgehenden Beschränkung der Gewalt auf Gegenstände und auf Auseinandersetzungen mit der Polizei scheint ein (idealtypisches) Charakteristikum von riots zu liegen, das sie von anderen Formen kollektiver Gewalt, etwa von Pogromen, unterscheidet. Allerdings schließt dies einerseits nicht aus, dass Menschen im Kontext oder am Rande von riots zu Schaden kommen und dass andererseits riots - dann häufig als race riots bezeichnet - existieren, in denen ethnisch motivierte Gewalt gegen Menschen eine Rolle spielen kann, so zum Beispiel bei den LA riots 1992 (vgl. allgemein zur Selektivität von Zielen im riot, auch hier mit einer Einschränkung in Bezug auf ethnic riots, della Porta/Gbikpi 2012: 89).

Im öffentlichen Diskurs dient die Bezugnahme auf die Gewaltförmigkeit von riots einerseits der Entpolitisierung solcher Ereignisse bzw. andererseits - da, wo sich politische Motive der Teilnehmenden nicht leugnen lassen - der Delegitimierung der politischen Anliegen. Welche Bedeutung der Gewalt im riot zugeschrieben oder zuerkannt wird, hängt aber wesentlich auch davon ab, was unter Gewalt verstanden wird, welcher Begriff oder welche Begriffe von Gewalt die Grundlage des Sprechens über sie sind.

Ein weites Gewaltverständnis dehnt den Begriff über die direkte physische Gewaltanwendung auf die den sozialen Verhältnissen innewohnende Gewalt aus. Für Johan Galtung umfasst die „strukturelle Gewalt“ all das, was eine potentiell mögliche menschliche Bedürfnisbefriedigung einschränkt (1969: 167ff.). Gegen den Begriff der strukturellen Gewalt könnte nun eingewandt werden, er mache die konkrete physische Gewaltanwendung ununterscheidbar von - sinnvollerweise als Macht und Herrschaft zu konzeptionierenden - sozialen Verhältnissen. Pierre Bourdieu unterscheidet hier zwischen konkretem physischen Gewalthandeln und einer „symbolischen 
Gewalt“. Diese „symbolische Gewalt“ versteht er als eine im Habitus verankerte Form der Gewalt, die die soziale Wirklichkeit als ein auf Herrschaft gegründetes Verhältnis unsichtbar zu machen trachte und sie auf diese Weise legitimiere (1997; 2012: 369ff., vgl. auch Krais/Gebauer 2010: 10; 52f.). Im Rückgriff auf weit gefasste Gewaltbegriffe kann die im riot zum Einsatz kommende Gewalt als eine gegen die gewaltvollen sozialen Verhältnisse gerichtete „Gegengewalt“ konzeptioniert werden (z. B. Fanon $1981 \mathrm{im}$ Kontext antikolonialer Befreiungskämpfe). Diese Konzeptionierung kann dann, je nach Standpunkt, als ideologisch motivierte Legitimierung eigenen Gewalthandelns verurteilt werden oder aber als sinnvolle Möglichkeit erscheinen, durch gesellschaftliche Herrschaftsverhältnisse hervorgebrachte oder provozierte Gewalt fassbar zu machen und sie zur Gewaltförmigkeit der gesellschaftlichen Strukturen in Beziehung zu setzen.

Enggeführte Gewaltbegriffe schränken das Verständnis von Gewalt demgegenüber auf die konkrete Anwendung physischer Gewalt ein. Dies muss nicht zwangsläufig, kann aber in bestimmten Fällen damit einhergehen, Fragen von Macht und Herrschaft in der Analyse von Gewalterscheinungen auszublenden oder gar als irrelevant zu erachten. In mikrosoziologischen Spielarten der Gewaltforschung wird - abstrahierend vom sozialen und politischen Kontext sowie von gesellschaftlichen Macht- und Herrschaftsverhältnissen - teilweise allein die Situation in den Blick genommen, die die Gewaltanwendung hervorzubringen scheint. Im Bestreben, alle Erscheinungsformen gewalttätigen Handelns von Menschen mit einem einzigen theoretischen Begriffsapparat beschreiben und erklären zu können, scheinen etwa bei Randall Collins (2011) die Gewalt der rioter und die ihnen typischerweise vorausgehende Polizeigewalt mit Todesfolge (aber auch kriegerische Konfrontationen, Gräueltaten und häusliche Gewalt) zumindest insofern das Gleiche zu sein, als dass sie im Rahmen einer einzelnen Theorie und eines einzelnen Kategoriensystems erfasst werden. Ein solches Vorgehen ist charakteristisch für die Gewaltforschung, welche die „Gewalt als solche“ als ihren zentralen Untersuchungsgegenstand begreift. Anders als andere Disziplinen, für die Gewalt nur einen Aspekt ihrer Untersuchungen darstellt, ist sie insofern legitimerweise bestrebt, ein theoretisches Modell zu entwerfen, mit dem sich verschiedenste Erscheinungsformen von Gewalt einordnen lassen. Dies gelingt im genannten Fall jedoch nur durch die weitgehende Ausblendung der Motive der Beteiligten und der zu Grunde liegenden sozialen Strukturen. Auf ähnliche Weise sehen etwa Paul und Schwalb unter anderem im ruandischen Genozid und den riots in England und Frankreich ,im Hinblick zwar nicht auf das Ausmaß, aber [...] auf die Dynamik nicht-organisierter kollektiver Gewalt vergleichbare Beispiele“ (2015: 7). Unter explizitem Verweis auf massenpsychologische Vorstellungen sehen sie bei diesen Ereignissen eine „Gewaltmasse“ als ein „besonderes Täterkollektiv“ (ebd.: 11) am Werk. Die Erklärung dieser Ereignisse wird in der Logik und Dynamik der Situation sowie in der Theoretisierung der „Masse“ als eines „ephemeren Zustand[s] körperlicher, affektiver und vorreflexiver Vergemeinschaftung" (Paul 2015: 57) gesucht (vgl. zum Begriff der Masse auch Günzel 2004, Gamper 2007).

Nun ist es weder illegitim, die Frage nach der spezifischen Beschaffenheit der Situation zu stellen, aus der heraus es zu einem riot kommt, noch ist es per se unberechtigt, nach der Rolle von Emotionen zu fragen oder danach, wie 
sich die Erfahrung kollektiven Handelns auf das Individuum auswirkt (zur Rolle von Emotionen bei kollektiver Gewalt und zur falschen Gleichsetzung von Emotionalität und Irrationalität im Kontext von riots vgl. Seferiades/ Johnston 2012a: 12f., vgl. allgemein auch das sub \urban-Themenheft „Gefühlsräume - Raumgefühle“ 2015). Im Gegenteil, ob und wie riots subjektkonstituierend wirken, ist eine oft vernachlässigte, aber entscheidende Frage (Trott 2014). In mikrosoziologischen Ansätzen - wie den oben genannten - können (und sollen) jedoch keine Aussagen darüber getroffen werden, wie riots überhaupt entstehen, von welchen sozialen Konfigurationen und Machtverhältnissen sie hervorgebracht und fortlaufend strukturiert werden. Für das Verständnis von riots geht der Erkenntnisgewinn solcher Ansätze über die Beschreibung von Regularitäten im Ablauf der konkreten Gewaltanwendung daher - so lautet unsere Einschätzung - nicht hinaus. Der mikrosoziologische Erkenntnisgewinn erfolgt - wie auch die Betonung vermeintlich rauschhafter Bewusstseinszustände und eines unreflektierten Handelns in der Masse - auf Kosten einer vorab vorgenommenen Entpolitisierung von riots.

Für uns steht außer Frage, dass der gesellschaftliche Kontext bzw. dass soziale Ungleichheit die Handlungen von Einzelnen oder Gruppen nicht unmittelbar determiniert. Solche „Hintergrundvariablen“ führen nicht automatisch zur Gewaltanwendung, und nicht jede_r strukturell Marginalisierte beteiligt sich an riots. Die Makroebene der gesellschaftlichen Verhältnisse aber komplett auszublenden oder zumindest als nachrangig zu betrachten (Collins 2011: 204f., Paul/Schwalb 2015: 10), erscheint uns allerdings als ebenso wenig zielführend, um das Phänomen zu verstehen. Um das Politische im riot fassen zu können, ist nicht notwendigerweise ein weiter Gewaltbegriff erforderlich, wohl aber ein offener Blick auf gesellschaftliche Verhältnisse, ein Blick also, der über die unmittelbare Situation der Gewaltanwendung hinausgeht (vgl. zur Frage des Politischen auch das sub \urban-Themenheft „Die postpolitische Stadt?" 2013). Auseinandersetzungen zwischen riotern und Polizei als eine gewalttätig verlaufende Interaktion unter vielen zu betrachten, verkennt die mit dem staatlichen Gewaltmonopol einhergehende Legitimation der Polizeigewalt, die jede Situation von riots im Sinne eines asymmetrischen Machtverhältnisses vorstrukturiert und für riots charakteristisch ist.

Charles Tilly geht davon aus, dass kollektive Gewalt, sobald Regierungen als Beobachter, Anspruchsteller oder Adressaten in Erscheinung treten, zu einem Spezialfall von contentious politics wird (2003: 9f.). Dabei nimmt er eine relationale Perspektive auf kollektive Gewalt ein. Diese grenzt er von einer auf Ideen und Überzeugungen verengten Sicht ab, aber vor allem auch von reduktionistischen verhaltensorientierten Perspektiven, die Impulse und Opportunitäten betonen (ebd.: $5 \mathrm{ff}$.). Auch ist Gewalt keineswegs das Gegenteil von Kommunikation. So schlägt etwa Daniel Briggs für die riots in Griechenland ein Konzept von „performativer Gewalt“ vor, die er als symbolisch expressiv fasst, insofern sie Forderungen und Ideen durch Gewalt zu kommunizieren und zu dramatisieren suche (2012: 366f.). Auch Fabien Jobard weist die Vorstellung zurück, rioter hätten keine Botschaft. Er betrachtet die Gewalt zudem als Mittel, um (teilweise durchaus erfolgreich) staatliche Ressourcen zu beanspruchen (vgl. dazu den Beitrag von Jobard und Greif in diesem Heft). 
In diesem Sinne erscheint die lang vorherrschende Annahme einer aufgebrachten Masse, die emotional und irrational zu ihrer eigenen Ungunst zur Gewalt greift und gebändigt werden muss, unzutreffend zu sein. Zudem versperrt sie die Sicht auf die Frage, warum rioting als Aktionsform auch das Ergebnis einer bewussten Entscheidung sein kann. Der Beobachtung, dass riots auch im Sinne eines progressiven Anliegens funktional sein können, trugen Frances Fox Piven und Richard Cloward bereits Ende der 1970er Jahre Rechnung. Sie argumentieren, dass disruptive action - also Handlungen, die Alltagsroutinen unterbrechen und normalisierte bzw. institutionalisierte Abläufe zum Stillstand bringen - entgegen der vorherrschenden Auffassung nicht nur rational, sondern auch strategisch einsetzbar und zielführend sein könnten; und zwar für diejenigen sozialen Gruppen, die wenig oder gar nicht von formalisierter Politik profitieren, sondern diese durchbrechen müssten, um ihren Anliegen überhaupt Gehör zu verschaffen (1979). In einem neueren Beitrag merkt Frances Fox Piven dazu an, dass ein Teil der Forschung seit den 1970er Jahren genau die gegenteilige Vereinfachung eingeschlagen habe. Die Protestbewegungen seien nun nicht mehr als irrational und damit als gefährlich wahrgenommen worden. Stattdessen sei die Gewalt, die manche Proteste begleitet, schlichtweg ignoriert oder allein den intervenierenden staatlichen Institutionen zugeschrieben worden (2012: 19). Piven bemerkt auch, dass die Analyse von kollektiver Gewalt als strategisch nicht ausschlieBen sollte, dass Handlungsformen wie riots auch einem expressiven bzw. emotionalen Zweck dienen könnten (ebd.: 24). Mit dieser Perspektive ist letztlich die Aufforderung verbunden, die Dichotomien rational-irrational und strategisch-emotional zu durchbrechen und genauer hinzuschauen, welche Beweggründe, Motivationen und Zielsetzungen riots jeweils zugrunde liegen. Dies erscheint sinnvoll, um einerseits der nicht zu leugnenden Rolle von Emotionen im Prozess des rioting gerecht werden zu können, und andererseits, um riots als (zumindest auch) strategisches Element im Raum des Politischen und als Protestform nicht aus dem Blick zu verlieren.

\section{Riot als Protest? Das Politische im rioting}

Wie Medien und politische Entscheidungsträger_innen riots interpretieren und wie die repressiven und sozialpolitischen Reaktionen aussehen, unterscheidet sich in den jeweiligen Ländern. Die Reduzierung von riots auf kriminelle Handlungen oder reinen Vandalismus ist dabei ein weit verbreitetes Muster. Nach den mehrtägigen riots in London im Sommer 2011 erklärte der britische Premier David Cameron, die Ereignisse hätten nichts mit Armut, sondern allein mit dem Verhalten der Beteiligten zu tun. Demnach hätte ein „kranker“ Teil der Gesellschaft jegliche moralischen Bezugspunkte verloren (Shipman 2011). Die harschen Verurteilungen und einseitigen Deutungen der Geschehnisse als individuelles, gar krankhaftes Fehlverhalten - also als gerade nicht durch gesellschaftliche Strukturen hervorgerufen - sind symptomatisch für die Aufarbeitung der riots in der englischen Presse und Öffentlichkeit (vgl. dazu den Beitrag von Marilena Simiti in diesem Heft). Dementsprechend waren auch die sozialpolitischen Maßnahmen infolge der riots äußerst marginal. Bereits vor den landesweiten riots in französischen Vorstädten im Oktober 2005 bezeichnete der damalige Innenminister 
Nicolas Sarkozy bei einem Besuch der Pariser banlieue-Stadt Argenteuil Vorstadtjugendliche als „Gesindel“, das man „mit dem Hochdruckreiniger“ (Zappi 2015) beseitigen müsse. Diese Äußerung heizte die ohnehin schon angespannte Situation in vielen Vorstädten weiter an. In der Debatte über die Ursachen der Aufstände verwies Sarkozy dann zwar auch auf Probleme wie Arbeitslosigkeit und Rassismus, bekräftigte jedoch im gleichen Atemzug seine vorherigen Äußerungen, es handele sich bei diesen Vorstadtjugendlichen um Kriminelle, die vor Gericht gestellt werden müssten. Wie Fabien Jobard im Interview in diesem Heft betont, gelang es den riotern jedoch durchaus auch, staatliche Investitionen in die Infrastruktur und die Förderung sozialer Projekte in den marginalisierten Vororten zu bewirken.

Eine Reihe von sozialwissenschaftlichen Veröffentlichungen widmete sich nach den Ausschreitungen in Frankreich, England, Griechenland und anderorts den Ursachen und politischen Dimensionen von riots. Im Folgenden stellen wir daher zwei politische Lesarten von riots in den Blickpunkt, die Ben Trott (2014) herausgearbeitet hat: Eine Lesart fokussiert den sozialpolitischen Kontext, in dem die riots stattfinden. Die andere Perspektive rückt die Frage ins Zentrum, inwiefern die rioter sich durch die Teilnahme am riot als politische Subjekte konstituieren.[5]

\section{Der sozialpolitische Kontext von riots}

Als eine Ursache für die riots in England, Frankreich und den USA wird von vielen Autor_innen die seit den $1980 e r$ Jahren wachsende soziale Ungleichheit benannt: Während das Bruttosozialprodukt und somit der durchschnittliche Wohlstand steigt, nimmt die sich auch räumlich niederschlagende Marginalisierung von bestimmten Bevölkerungsgruppen zu (Wacquant 2006: 23). Hierfür wird vor allem eine neoliberale Wirtschaftspolitik verantwortlich gemacht (siehe Dzudzek/Müller 2013, Slater 2011, Sotiris 2010, Trott 2014), die Maßnahmen der finanz- und kapitalwirtschaftlichen Deregulierung, (Teil-) Privatisierung staatlicher Unternehmen sowie des öffentlichen Dienstes und die Reduzierung von staatlichen Sozialleistungen umfasst. Quantitative Daten bestätigen diese These: Thomas Piketty (2016) weist nach, dass die Bedeutung von Kapitalvermögen spätestens seit den 1970er Jahren in allen Ländern Westeuropas und Nordamerikas wieder zunimmt. In diesen Ländern macht das Privatkapital mittlerweile zwischen 400 und 600 Prozent des Nationaleinkommens aus, wobei Piketty eine weiter steigende Tendenz prognostiziert. Weil die Kapitalrendite höher als das Wirtschaftswachstum und der Einkommenszuwachs ist, werden Kapitalbesitzer_innen stets reicher und das Vermögen konzentriert sich immer stärker. Die Kluft zwischen Arm und Reich vergrößert sich also zunehmend.[6] Zugleich verfügen immer weniger (vor allem junge) Menschen über ein geregeltes Einkommen. Die Jugendarbeitslosigkeit lag in den von riots betroffenen Stadtgebieten in Frankreich, in Großbritannien und in den USA weit über dem jeweiligen nationalen Durchschnitt.[7] In Griechenland stieg die Jugendarbeitslosigkeit zwischen 2008 und 2012 von 22,1 auf 55,2 Prozent (OECD 2013).

Balibar sieht die entscheidende Transformation hin zu einer neoliberalen Wirtschaftsordnung darin, dass die Bedingungen von Klassen nicht mehr über die Arbeitsteilung, sondern über die Spaltung zwischen relativ sicheren und 
prekären Jobs definiert würden. Da die Löhne nicht mehr vor Armut bewahren und viele Arbeitnehmer_innen keinen gewerkschaftlichen Schutz mehr genießen, werde der Lohnarbeitsvertrag, wie es Wacquant ausdrückt, „selbst zu einer Quelle von Fragmentierung und Unsicherheit“ (2006: 25f.). Darüber hinaus werde durch den Wandel der Arbeitswelt ein großer Teil der erwerbstätigen Bevölkerung überflüssig (ebd.: 25). Insofern erschienen manche banlieues als „Wohnräume für ein neues Proletariat, dessen Unsicherheit maximal ist und dem nur die Wahl zwischen unsicherer Arbeit und Arbeitslosigkeit bleibt“ (2007: 58, eigene Übersetzung, Hervorhebung im Original).

Die Frage nach dem politischen Gehalt von riots bzw. rioting in marginalisierten Stadtvierteln lässt sich nicht beantworten, ohne den diskriminierenden und konfliktiven Alltag zu betrachten. In vielen Arbeiten wird der Zusammenhang zwischen sozialökonomischer Ungleichheit und Rassismus als Ursache für riots herausgearbeitet (Balibar 2007, Waddington/ King 2012). Insbesondere das Verhältnis zwischen Jugendlichen und Polizei ist hier von grundlegender Bedeutung. In den meisten Fällen ist der Auslöser für riots eine eskalierende Konfrontation zwischen Jugendlichen und Polizei. Vor dem Hintergrund einer erhöhten Polizeipräsenz sowie einer zunehmend repressiv ausgerichteten Sicherheitspolitik (Fassin 2011, Garland 2008, Wacquant 2009) scheinen männliche Jugendliche mit familiärem Migrationshintergrund von Polizeikontrollen - sowohl in marginalisierten Stadtvierteln als auch in urbanen Zentren - am stärksten betroffen zu sein. Das ist eine oft betonte, zugleich aber wenig erforschte Beobachtung (Greif 2012). Der konfrontative Kontakt mit der Polizei ist für die Jugendlichen oft alltäglich. Dass sich die Aufmerksamkeit einer überwiegend weißen männlichen Polizei (Jobard 2008, hier bezogen auf den französischen Kontext) verstärkt auf männliche Jugendliche als Kollektivadressat polizeilicher Handlungslogik (vgl. Behr 2003: 195ff.) richtet, erscheint wenig verwunderlich, da die Polizei an der medialen Konstruktion von gefährlichen bzw. gefährdeten Jugendlichen als „dramatisierender Experte“ selbst aktiv beteiligt ist (Behr 2003; 2006, Jobard 2008; 2015). Neben einer Ethnisierung von Delinquenz und dem racial profiling, spielen für die diskriminierende Etikettierung von „verdächtigen“Jugendlichen durch die Polizei auch territoriale Qualifizierungsmerkmale eine Rolle (Jobard 2008). Diese „Ortseffekte“ (Bourdieu 2002) marginalisierter Stadtviertel sind ebenfalls Bestandteil einer rassistischen Polizeipraxis gegenüber Jugendlichen. Aus einer alltagsorientierten konfliktsoziologischen Perspektive betrachtet, handelt es sich bei riots bzw. rioting folglich um Eskalationen eines alltäglichen Konfliktes zwischen Jugendlichen und Polizei. Allerdings führt dieses „InSchach-Halten durch Strafe“ (Wacquant 2009: 51f.) als staatliche Technik, um sich verschärfende Marginalität zu managen, nicht bei jedem Kontakt mit der Polizei zu größeren Ausschreitungen. In den meisten Fällen eskaliert eine Konfrontation mit der Polizei dann, wenn Jugendliche dabei zu Tode kommen oder schwer verletzt werden.

Bei der Analyse der verschiedenen gesellschaftlichen Kontexte, aus denen riots hervorgehen, wird deutlich, dass es sich bei den ethnisierten Gruppen in Frankreich um andere Teilnehmer_innen handelt als beispielsweise bei den Antiglobalisierungsprotesten in Deutschland, Italien und Griechenland. Bei letzteren lassen sich die Protestierenden überwiegend dem weißen 
Mittelstand und anarchistischen sowie anderen linksradikalen Kreisen zuordnen (Thompson 2010, Seferiades/Johnson 2012b, Martinez 2004). Ohne die sozialpolitischen Hintergründe und konkreten Ausgrenzungserfahrungen gleichsetzen zu wollen, lässt sich fragen, warum rioter mit unterschiedlichen sozioökonomischen Hintergründen und Marginalisierungserfahrungen zu einem ähnlichen Ausdrucksmittel greifen, bei dem die „institutionalisierten juristisch-politischen Regeln sozialer Konfliktaustragung“ (Mücke/Rinn in diesem Heft) kollektiv ausgesetzt werden. Wie einige Veröffentlichungen zu den Ereignissen in England und Frankreich betonen, wird insbesondere in marginalisierten Stadtvierteln den riots ein politischer Charakter abgesprochen (siehe hierzu den Beitrag von Simiti in diesem Heft). Soziale Strukturen werden zwar als Ursachen anerkannt, die Ausschreitungen selbst aber meist als irrationale Gewalt gedeutet. Beim rioting eines black bloc[8] werden wiederum sozialstrukturelle Ursachen für das Handeln der Beteiligten fast immer ausgeschlossen, vielmehr werden dafür eine fehlgeleitete und destruktive politische Ideologie verantwortlich gemacht. Zu fragen ist jedoch, ob diese scharfe Trennung zwischen sozialen und politischen Beweggründen tatsächlich weiterführt. Lässt sich die auf die französischen banlieues bezogene These von Balibar, dass es sich bei den riots um einen Akt zur Erlangung von Repräsentation, Anerkennung und Partizipation handelt (2007: 58), nicht auch auf andere Kontexte des rioting beziehen?

Wie in Texten zu anarchistischen und linken riots deutlich wird (Thompson 2010, Seferiades/Johnston 2012b, Sotiris 2010), sind es nicht nur die klassisch Marginalisierten, die gegen Prekarisierung protestieren (Dörre 2015: 39). Auch die Mittelschicht in Europa und in den USA ist zunehmend mit einer steigenden sozialen Ungleichheit konfrontiert, denn weder Mittelschichtszugehörigkeit noch akademischer Bildungsgrad schützen heute vor unsicheren Arbeits- und Lebensverhältnissen (Dörre 2015: 68). Auch ist das Vertrauen in die repräsentative Demokratie nicht nur bei politischen Aktivist_innen stark geschwächt, vielmehr handelt es sich um ein allgemeines gesellschaftliches Phänomen, wie der Mitgliederschwund der etablierten Parteien nahelegt (siehe etwa für Griechenland Seferiades/Johnston 2012b: 150). Dementsprechend wird die Sprach- und Forderungslosigkeit, die als Charakteristikum für die riots in Frankreich und England angesehen wird (Castel 2009: 56), oft mit einem Repräsentationsdefizit begründet (auch Balibar 2007: 62). Während es in den 1980er Jahren noch soziale Organisationen in den betroffenen Stadtvierteln gab, die für ihre Bewohner_innen auch repräsentative Funktionen ausübten, änderte sich dies im Zuge einer zunehmenden Neoliberalisierung von Wirtschaft und Gesellschaft (vgl. Dzudzek/Müller 2013: 27). Doch riots werden nicht nur als Ausdruck einer Repräsentationskrise gewertet, sondern als Aufstand gegen eine Politik der Repräsentation (Altenried 2012: 53, ähnlich Dzudzek/Müller 2013: 31). Riots stellen, auf diese Weise interpretiert, eine „bewusste Absage an eine Mehrheitsgesellschaft und an ein politisches System“ dar, „von dem sich viele der Aufständischen nichts mehr erhoffen“ (Altenried 2012: 53). Vor diesem Hintergrund erscheint die Zukunft wenig hoffnungsvoll, sondern ausweglos, unsicher und prekär. Slater resümiert entsprechend: „[W]enn junge Menschen der Wahrnehmung beraubt werden, dass vor ihnen eine würdevolle Zukunft liegt, werden sie in kollektiver Wut auf die Straße gehen." (2011: 109, eigene Übersetzung) 


\section{Die subjektkonstituierende Wirkung von riots}

Die Auseinandersetzungen mit riots beschränken sich nicht darauf, politische und soziale Ursachen herauszuarbeiten. Manche Autor_innen (z. B. Balibar, Trott und Altenried) betonen, dass riots in marginalisierten Stadtteilen nicht nur als Symptom gesellschaftlicher Missstände, sondern auch als politische Subjektartikulation gelesen werden sollten. Riots sind demnach „ein Moment (wie fragil, unsicher, umkehrbar dieser auch sein mag), in dem sich ein politischer Prozess entfaltet (...), der zum Bewusstsein und Handeln derjenigen gehört, die seine Träger sind“ (Balibar 2007: 61, eigene Übersetzung). Auch Dzudzek und Müller sprechen von riots als Aufhebung des „normalisierten Ausnahmezustands“, wodurch sich kurzzeitig politische Subjekte neu formierten, sich allerdings nicht langfristig etablieren könnten (2013: 32). Altenried sieht in den englischen riots hingegen deutliche Ansätze für ein neues politisches Bewusstsein. Die riots hätten ein „kollektives Gefühl des Widerstands" und neue Solidaritäten geschaffen sowie alte gefestigt (2012: 51). Thompson formuliert die These noch eindrücklicher, wenn er behauptet, dass Gewalt immer ein Faktor in der Genese neuer politischer Subjektivität gewesen sei (2010: 132). Er folgert, dass weiße mittelständische Aktivist_innen durch die riots bei den Antiglobalisierungsprotesten wieder in den Bereich des Politischen getreten seien und sich nicht mehr mit dem Bestehenden arrangiert und einem reinen Selbstmanagement überlassen hätten. Riots böten in dieser Logik die Möglichkeit, die etablierte Form der Politik, nämlich jene der Repräsentation, zu überschreiten und so in einen post-repräsentativen Zustand der Politik einzutreten (ebd.:133).

Den hier wiedergegebenen Thesen zur politischen Subjektivität von riotern ist gemeinsam, dass der riot keine politische Subjektivität voraussetzt, sondern diese erzeugt. Zu fragen bleibt dennoch, ob riots wirklich mit einer politischen Subjektwerdung einhergehen und nicht nur das Gefühl von Kollektivität und Authentizität vermitteln. Wie lässt sich aber der politische Gehalt von Handlungen (methodisch) erfassen, wenn sie sich traditionellen Protestformen entziehen? Die bisherige Forschung gibt auf diese Fragen nur unzureichende Antworten. Wir sind überzeugt davon, dass, um riots als Protestform besser zu verstehen und einordnen zu können, noch viele Fragen gestellt und beantwortet werden müssen. Die Soziale Bewegungsforschung bietet mit ihren analytischen Instrumenten und Begrifflichkeiten nur begrenzte Möglichkeiten, riots in all ihren Facetten zu betrachten, da sie sich häufig nur Bewegungsorganisationen untersucht. Daher wird die „Analyse expressiver und eruptiver Formen von Protest“ sogar als Blindstelle der Bewegungsforschung bezeichnet (Teune 2008: 541). Vor dem Hintergrund einer zunehmenden ökonomischen Ungleichheit und Marginalisierung groBer Bevölkerungsteile stellt sich die Frage, ob Protest, der nicht mehr durch traditionelle Mechanismen der Repräsentation aufgefangen werden kann, entsprechend neue bzw. andere analytische Zugänge erfordert.

Wir hoffen, dass die Texte in diesem Themenheft dazu beitragen, eine solche Richtung einzuschlagen. Die Vielfalt der empirischen Beispiele und der analytischen Perspektiven in diesem Heft verweisen darauf, wie weit das thematische Feld riots ist und wie viele lohnenswerte Anknüpfungspunkte es für weitere Forschungen gibt. So zeigen Yannick Kalff und Katharina Warda in 
ihrem Text über die Chaostage in Hannover (1995), wie die breite Öffentlichkeit jene riots als „stupide, sinnlose Unruhe“ wahrgenommen und auf individualisierende und psychologisierende Erklärungsmuster zurückgegriffen hat. Eine ähnliche Depolitisierung und Bagatellisierung konstatieren auch Julika Mücke und Moritz Rinn in ihrem Artikel über die Konfrontationen zwischen Polizei und einer Gruppe ethnisierter Jugendliche in Hamburg-Altona. Während die Konflikte in den meisten Medien primär auf die hohe Kriminalität, den gefährlichen Charakter des Stadtviertels und die dort lebenden frustrierten und aggressiven Jugendlichen zurückführt wurden, argumentieren die Autor_innen, dass es bei den Auseinandersetzungen vielmehr um eine bestimmte Ausprägung von Rassismus und spezifische Polizeistrategien ging. Anders als die Ereignisse in Hamburg-Altona werden die riots von 2008 und 2011 in Griechenland, wie Marilena Simiti und Dilan Köse in ihren Beiträgen in diesem Heft darstellen, dagegen durchaus als politische Handlungsform interpretiert. Weite Teile der Bevölkerung teilen das Verständnis der riots als legitime sozialen Revolte. Schließlich untersucht Bettina Engels in ihrem Text, wie etablierte Bewegungsakteur_innen in Burkina Faso sich auf food riots beziehen, um ihre eigenen Forderungen zu legitimieren.

\section{Endnoten}

[1] Vgl. z. B. Raschke 1987, Roth/Rucht 2008. So erklärt Raschke im Kontext seiner Abgrenzung von sozialen Bewegungen und „kollektiven Episoden“, „die Kategorie des ,sozialen Protests““ sei „für die Bewegungsforschung nicht sehr hilfreich. Sie verwischt den hier wichtigen Unterschied zwischen Aktivitäten stärker strukturierter Kollektive, mit denen wir es bei sozialen Bewegungen zu tun haben, und schwach strukturierter Kollektive, die sich bei ad hoc-Aktivitäten zusammenfinden“ (1987: 79).

[2] Siehe hierzu z. B auch Bayat 2010. In seiner Analyse zu den Ländern des Nahen Ostens arbeitet Bayat heraus, dass subversive Alltagspraktiken von Subalternen eine Form von Protestartikulation darstellen, die die Bewegungsforschung nicht fassen kann und die er deshalb „social non-movements“ nennt.

[3] Das Strafgesetzbuch für das Deutsche Reich vom 15. Mai 1871, nach der Novelle vom 26. Februar 1876, nebst Einführungs- und Abänderungsgesetz, 34-35. https://archive.org/ stream/dasstrafgesetzboogermgoog\#page/n44/mode/2up (letzter Zugriff am 19.3.2016).

[4] Public Order Act 1986, Chapter 64, Part 1(1). http://www.legislation.gov.uk/ ukpga/1986/64 (letzter Zugriff am 19.03.2016).

[5] Trott schlägt noch eine dritte politische Lesart vor, die sich darauf bezieht, wie riots zum Objekt von Politik werden (2014).

[6] Beispielsweise besaßen in Großbritannien - dem Land, in dem die Schere zwischen Arm und Reich in Europa am weitesten auseinander ging - die reichsten 0,1 Prozent der Bevölkerung 1970 circa 1,2 Prozent des Gesamtvermögens. 2005 war dieser Prozentsatz auf 5 Prozent gestiegen (Dzudzek 2013: 26).

[7] Die Jugendarbeitslosigkeit in französischen Vororten liegt im Durschnitt bei 40 Prozent (Bareis/Bojadzijev: 846) und auch die von riots betroffenen Stadtviertel in England weisen eine erhöhte Jugendarbeitslosigkeit auf (Sutterlüty 2014: 41). In der Metropolenregion von St. Louis (also der Stadt, zu der Ferguson gehört) sind 47 Prozent der afroamerikanischen Jugendlichen arbeitslos (Trasher 2014).

[8] Mit black bloc oder auch „Schwarzer Block“ bezeichnet man anarchistische und/oder antifaschistische Gruppen, die auf Demonstrationen oder Protestveranstaltungen überwiegend schwarz gekleidet auftreten und in militanter Weise agieren. Gewaltvolle Auseinandersetzungen mit der Polizei oder auch Sachbeschädigung gehören häufig zum Mittel ihrer Wahl. 


\section{Autor_innen}

Janna Frenzel ist Politikwissenschaftlerin und forscht zu Narrativen über die Jugendaufstände in Stockholm 2013.

jannafrenzel@gmx.de

Philippe Greif ist Soziologe mit Interesse an urbaner Marginalität, Ethnographie, Intersektionalität/sozialer Ungleichheit und Geschlechterforschung. Er forscht aktuell zum Alltag von Jugendlichen in Pariser Banlieues.

phi.greif@googlemail.com

Fabian Klein ist Soziologe, beschäftigt sich schwerpunktmäßig mit sozialer Ungleichheit und forscht derzeit zu Diskursen während der English riots in England 2011.

fabian.klein-f44@ruhr-uni-bochum.de

Sarah Uhlmanns Forschungsinteressen liegen im Bereich der städtischen sozialen Bewegungen und der politischen Ökonomie.

sarah.uhlmann@hu-berlin.de

\section{Literatur}

Altenried, Moritz (2012): Aufstände, Rassismus und die Krise des Kapitalismus. England im Ausnahmezustand. Münster: edition assemblage.

Balibar, Étienne (2007): Uprisings in the Banlieues. In: Constellations 14, 47-71.

Bareis, Ellen / Bojadzijev, Manuela (2010): Jenseits von Forderungen und Organisierung. Revolten in den französischen Vorstädten. In: Das Argument 52/289, 839-848.

Behr, Rafael (2003): Die Polizei als Konstrukteur adoleszenter Konformität und Abweichung. In: DVJJ (Hg.), Jugend, Gesellschaft und Recht im neuen Jahrtausend. Blick zurück nach vorn; Dokumentation des 25. Deutschen Jugendgerichtstages vom 28. September bis 2. Oktober 2001 in Marburg. Mönchengladbach: Forum Verlag Godesberg, 185-205.

Behr, Rafael (2006): Polizeikultur. Routinen - Rituale - Reflexionen. Bausteine zu einer Theorie der Praxis der Polizei. Wiesbaden: VS Verlag für Sozialwissenschaften / GWV Fachverlage GmbH, Wiesbaden.

Bourdieu, Pierre (1997): Eine sanfte Gewalt. Pierre Bourdieu im Gespräch mit Irene Dölling und Margareta Steinrücke. In: Irene Dölling / Beate Krais (Hg.), Ein alltägliches Spiel. Frankfurt am Main: Suhrkamp, 218-230.

Bourdieu, Pierre (2002): Ortseffekte. In: Pierre Bourdieu (Hg.), Das Elend der Welt. Zeugnisse und Diagnosen alltäglichen Leidens an der Gesellschaft. [Nachdr.]. Konstanz: UVK Verlagsgesellschaft (Édition discours, 9), 159-167.

Bourdieu, Pierre (2012): Entwurf einer Theorie der Praxis auf der ethnologischen Grundlage der kabylischen Gesellschaft. Frankfurt am Main: Suhrkamp.

Briggs, Daniel (2012): Post-modern Greek Tragedy: Walking in the Steps of Thucydides in Athens. In: Daniel Briggs (Hg.), The English Riots of 2011. A Summer of Discontent. London: Waterside Press, 361-379.

Castel, Robert (2009): Negative Diskriminierung: Jugendrevolten in den Pariser banlieues. Hamburg: Hamburger Ed.

Collins, Randall (2011): Dynamik der Gewalt. Eine mikrosoziologische Theorie. Hamburg: Hamburger Edition.

della Porta, Donatella / Diani, Mario (1999): Social Movements. An Introduction. Second Edition. Malden / Oxford / Carlton: Blackwell Publishing.

della Porta, Donatella / Gbikpi, Bernard (2012): The Riots: A Dynamic View. In: Seraphim Seferiades / Hank Johnston (Hg.), Violent Protest, Contentious Politics, and the Neoliberal State. Farnham / Burlington: Ashgate, 87-100.

Dikeç, Mustafa (2004): Voices into noises: ideological determination of unarticulated justice movements. In: Space and Polity 8/2, 191-208.

Dörre, Klaus (2015): Génération Précaire - ein europäisches Phänomen. In: Michael Busch / Jan Jeskow / Rüdiger Stutz (Hg.), Zwischen Prekarisierung und Protest: Die Lebenslagen und Generationsbilder von Jugendlichen in Ost und West. Bielefeld: transcript Verlag, 39-74. 
Dzudzek, Iris / Müller, Michael (2013): Der Lärm des Politischen. Die Londoner riots 2011 und ihre politischen Subjekte. In: sub \urban. zeitschrift für kritische stadtforschung, Band 2 Heft 1, 17-40.

Fanon, Frantz (1981): Die Verdammten dieser Erde. Frankfurt a. M.: Suhrkamp.

Galtung, Johan (1969): Violence, peace and peace research. In: Journal of Peace Research $6 / 3,167-191$.

Fassin, Didier (2011): La force de l'ordre. Une anthropologie de la police des quartiers. Paris: Éditions du Seuil.

Gamper, Michael (2007): Masse lesen, Masse schreiben. Eine Diskurs- und Imaginationsgeschichte der Menschenmenge 1765-1930. München: Wilhelm Fink Verlag.

Garland, David (2008): Kultur der Kontrolle. Verbrechensbekämpfung und soziale Ordnung in der Gegenwart. Frankfurt a. M., New York: Campus.

Guardian / LSE (2011): Reading the Riots: Investigating England's Summer of Disorder. London: Guardian / LSE.

Günzel, Stephan (2004): Der Begriff, Masse“ in Philosophie und Kulturtheorie. In: Dialektik 2004/2, 117-135.

Greif, Philippe (2012): London Calling Paris. Zur Relevanz der Kategorie Männlichkeit für eine intersektionale Analyseperspektive auf urbane Jugendaufstände in Europa. http:// www.fu-berlin.de/sites/gpo/soz_eth/Frauen-_M_nnerforschung/London_Calling Paris_Zur_Relevanz_der_Kategorie_M_nnlichkeit_f_r_eine_intersektionale_ Analyseperspektive_auf_urbane_Jugendaufst_nde_in_Europa/index.html (letzter Zugriff: 27.03.2016).

Jobard, Fabien (2008): Ethnizität und Rassismus in der gesellschaftlichen Konstruktion der gefährlichen Gruppen. Polizeikultur und -praxis in den französischen Vororten. In: Schweizerische Zeitschrift für Soziologie 34/2, 261-280.

Jobard, Fabien (2015): Police et Jeunesse. In: Idées économiques et sociales 181, 40-47.

Keith, Michael (1993): Race, Riots and Policing: Lore and disorder in a multi-racist society. London: UCL Press.

Krais, Beate / Gebauer, Gunter (2002): Habitus. Bielefeld: transcript.

Martinez, Elizabeth Bettina (2004): Where Was the Color in Seattle? Looking for reasons why the Great Battle was so white. In: Monthly Review 52, 141-148.

Marx, Gary T. (1970): Issueless Riots. In: Annals of the American Academy of Political and Social Science 391, 21-33.

Mennel, Birgit / Nowotny, Stefan (Hg.) (2014): Die Sprachen der Banlieues. Wien: transversal texts.

OECD (2013): Employment and labour markets: Key tables from OECD. http://www.oecdilibrary.org/employment/youth-unemployment-rate_20752342-table2 (letzter Zugriff am 21.03.2016).

Paul, Axel T. (2015): Masse und Gewalt. In: Axel T. Paul / Benjamin Schwalb (Hg.), Gewaltmassen. Über Eigendynamik und Selbstorganisation kollektiver Gewalt. Hamburg: Hamburger Edition, 19-59.

Paul, Axel T. / Schwalb, Benjamin (Hg.) (2015): Gewaltmassen. Über Eigendynamik und Selbstorganisation kollektiver Gewalt. Hamburg: Hamburger Edition.

Phillips, Richard / Frost, Diane / Singleton, Alex (2013): Researching the Riots. In: The Geographical Journal 179/1, 3-10.

Piketty, Thomas (2016): Das Kapital im 21. Jahrhundert. München: C.H.Beck.

Piven, Frances Fox (2012): Protest Movements and Violence. In: Seraphim Seferiades / Hank Johnston (Hg.), Violent Protest, Contentious Politics, and the Neoliberal State. Farnham / Burlington: Ashgate, 19-28.

Piven, Frances Fox / Cloward, Richard A. (1979): Poor People's Movements. Why they succeed, how they fail. New York: Vintage Books.

Raschke, Joachim (1987): Soziale Bewegungen. Ein historisch-systematischer Grundriss. Frankfurt a. M. / New York: Campus.

RIAD RuhrgebietsInternationalismusArchiv Dortmund (2012): „Das Leben ist eine Demo, Frankreich eine Glasscheibe und ich bin ein Pflasterstein“. Versuch über das Verhältnis von Rap und Revolte. In: Kollektiv Rage: Banlieues. Die Zeit der Forderungen ist vorbei. Berlin / Hamburg: Assoziation A, 207-225.

Roth, Roland / Rucht, Dieter (Hg.) (2008): Die sozialen Bewegungen in Deutschland seit 1945. Ein Handbuch. Frankfurt am Main / New York: Campus. 
Seferiades, Seraphim / Johnston, Hank (2012a): The Dynamics of Violent Protest: Emotions, Repression and Disruptive Deficit. In: Seraphim Seferiades / Hank Johnston (Hg.), Violent Protest, Contentious Politics, and the Neoliberal State. Farnham / Burlington: Ashgate, 3-18.

Seferiades, Seraphim / Johnston, Hank (2012b): The Greek December. In: Seraphim Seferiades/Hank Johnston (Hg.), Violent Protest, Contentious Politics, and the Neoliberal State. Farnham / Burlington: Ashgate, 149-156.

Shipman, Tim (2011): Parts of our society are not broken but sick - the Prime Minister's verdict on Riot UK. http://www.dailymail.co.uk/news/article-2024709/David-CameronUK-riots-Parts-society-broken-sick.html (letzter Zugriff am 28.03.2016).

Slater, Tom (2011): From 'Criminality' to Marginality: Rioting Against a Broken State. In: Human Geography 4/3, 106-15.

Sotiris, Panagiotis (2010): Rebels with a Cause: The December 2008 Greek Youth Movement as the Condensation of Deeper Social and Political Contradictions. In: International Journal of Urban and Regional Research 34, 203-209.

sub \urban (2013): Die postpolitische Stadt? Themenheft, sub \urban. zeitschrift für kritische stadtforschung, Band 1 Heft 2. herausgegeben von Boris Michel und Nikolai Roskamm.

sub \urban (2014): Illegalität - Stadt - Polizei. Themenheft, sub \urban. zeitschrift für kritische stadtforschung, Band 2 Heft 2.

sub \urban (2015): Gefühlsräume - Raumgefühle. Themenheft, sub\urban. zeitschrift für kritische stadtforschung, Band 3 Heft 2, herausgg. von Benno Gammerl und Rainer Herrn.

Sutterlüty, Ferdinand (2014): The hidden morale of the 2005 French and 2011 English riots. In: Thesis Eleven 121, 38-56.

Teune, Simon (2008): „Gibt es so etwas überhaupt noch?“ Forschung zu Protest und sozialen Bewegungen. In: Politische Vierteljahresschrift 49, 528-547.

Thompson, A.K. (2009): Black Bloc, White Riot: Antiglobalization and the Genealogy of Dissent. Edinburgh: AK Press.

Tilly, Charles (2003): The Politics of Collective Violence. Cambridge: Cambridge University Press.

Tilly, Charles / Wood, Lesley J. (2013): Social Movements, 1768-2012. Third Edition. London / New York: Routledge.

Tilly, Charles / Tarrow, Sidney (2015): Contentious Politics. Second Edition. New York: Oxford University Press.

Trasher, Steven W. (2014): The real looting of Ferguson: its black citizens never had a chance to get by. http://www.theguardian.com/commentisfree/2014/aug/19/ferguson-lootingnational-guard-black-citizens-get-by (letzter Zugriff am 21.03.2016).

Trott, Ben (2014): Research and the Riots: Politics and England's 2011 Urban Uprisings. http://councilforeuropeanstudies.org/critcom/research-and-the-riots-politics-andenglands-2011-urban-uprisings (letzer Zugriff am 10.03.2016).

Wacquant, Loïc (2006): Das Janusgesicht des Ghettos und andere Essays. Basel: Birkhäuser Verlag.

Wacquant, Loïc (2009): Bestrafen der Armen. Zur neoliberalen Regierung der sozialen Unsicherheit. Opladen / Berlin / Toronto: Verlag Barbara Budrich.

Waddington, David / Jobard, Fabien / Mike King (Hg.) (2009): Rioting in the UK and France. A comparative analysis. Cullompton: Willan Publishing.

Waddington, David / King, Mike (2012): Contemporary French and British Urban Riots : An Exploration of the Underlying Political Dimensions. In: Seraphim Seferiades / Hank Johnston (Hg.), Violent Protest, Contentious Politics, and the Neoliberal State. Farnham / Burlington: Ashgate.

Zappi, Silvia (2015) : Dix ans après, que reste-t-il des émeutes urbaines de 2005? http:// www.lemonde.fr/societe/article/2015/o3/16/dix-ans-apres-que-reste-t-il-des-emeutesurbaines-de-2005_4594328_3224.html?xtmc=sarkozy_racailles_2005\&xtcr=1 (letzter Zugriff am 27.03.2016). 